\title{
TEXTO
}

\section{EL DIARIO DE ALEXANDER VON HUMBOLDT EN ESPAÑ}

\author{
Ulrike Leitner \\ Berlin-Brandenburgische Akademie der Wissenschaften
}

\section{RESUMEN}

El diario español de Humboldt se encuentra sorprendentemente en una carpeta de su legado en Berlín, donde el mismo recopiló los materiales para su publicación sobre la meseta española en la revista Hertha, y no en sus diarios encuadernados del viaje americano. Esto explica que no se hubieran descubierto hasta hace poco tiempo. El manuscrito contiene descripciones geognósticas, observaciones sobre el clima, la vegetación, la electricidad de la atmósfera, etc. La comparación con lo publicado de Humboldt en 1825 sobre la meseta española que realizamos en las notas de esta edición facilitará al lector una aproximación a los métodos cientificos típicos de Humboldt.

PALABRAS CLAVE: Manuscritos. Historia de las Ciencias Naturales. Mediciones geodésicas. Geología. Legado de Humboldt. Clima. Vegetación. Electricidad de la atmósfera.

\section{THE JOURNAL OF A. V. HUMBOLDT IN SPAIN}

\section{ABSTRACT}

The Spanish diary can surprisingly not be found in his bounded diaries from his American travels, but in a separate folder of his legacy in Berlin, where Humboldt collected material which he wanted to use for his publication about Spain. So it remained undiscovered until recently. Humboldt's notes contain geognostic descriptions as well as his observations about climate, vegetation, electricity of the atmosphere, etc. The comparison with Humboldt's publication of 1825 facilitates to gain insight into his scientific methods.

* Traducción, a cargo de María Rosario Martí Marco, de LeITner, U. (2007), Alexander von Humboldts spanisches Tagebuch, Berliner Manuskripte zur Alexander-von-HumboldtForschung, 28, Berlin, Alexander-von-Humboldt-Forschungsstelle. 
KEY WORDS: Manuscripts. History of Natural Sciences. Geodesic measurements. Geology. The Humboldt papers. Climate. Vegetation. Electricity of the atmosphere.

\section{INTRODUCCIÓN}

Por fin, pues, rumbo a España

Se suele señalar como inicio del viaje americano de Humboldt el día de la partida desde La Coruña en el barco Pizarro al 5 de junio, aunque realmente comenzó anteriormente, en París. En consecuencia, la edición de los diarios ${ }^{1}$ empieza, en el primer capítulo, con la descripción del trayecto de París a Toulon.

Humboldt relata en las primeras páginas los últimos preparativos, la despedida de su hermano y los planes del viaje. El 20 de octubre de 1798 abandona París acompañado por Aimé Bonpland. El recorrido en silla de posta les conducirá a Mâcon, adonde llegaron el 23 de octubre, y continuaron al día siguiente en barca por el río Saône hasta llegar a Lyon, donde reanudaron la ruta en silla de posta con dirección a Marsella. Allí esperaron en vano, hasta mediados de diciembre, la posibilidad de llegar al norte de África, y en este intervalo de tiempo Humboldt y Bonpland realizaron diversas excursiones, una de ellas a Toulon, de varios días de duración. Por fin el 15 de diciembre partieron hacia España.

Desde el 8 hasta el 28 de enero de 1799 los dos viajeros permanecieron en Barcelona y realizaron una excursión a Montserrat. Continuaron el viaje en paralelo al Mediterráneo hasta llegar a Valencia, donde estuvieron hasta el 8 de febrero. Desde aquí los viajeros se dirigieron a Madrid, adonde llegaron probablemente el 23 de mayo.

En Madrid, Humboldt redactó varias cartas en las que daba cuenta del viaje realizado hasta ese momento: «Hice la mayor parte del viaje a pie, a lo largo de la costa mediterránea, pasando por Cette, Montpellier, Narbonne, Perpiñán, los Pirineos y Cataluña hasta Valencia y Murcia, atravesando después la meseta de La Mancha, hasta llegar aquí», escribía Humboldt a Willdenow desde Madrid. «Mientras en los valles pirenaicos prosperaban las legumbres, El Canigó eleva su cumbre cubierta de nieve. En Cataluña y en Valencia la tierra es un eterno jardín, adornado con cactus y pitas. Las palmeras de dátiles, de 40 a 50 pies de altura, cargadas de racimos de frutos, sobrepasan

1 FAAK, M. (ed.) (2000), Alexander von Humboldt. Reise durch Venezuela. Auswahl aus den amerikanischen Reisetagebüchern, Beiträge zur Alexander-von-Humboldt-Forschung, 12, Berlin, Akademie Verlag, pp. 43-56. 
la altura de los monasterios. [...] La campiña reverdecía y en la tierra yerma recogimos narcisos y junquillos (jonquillen). [...] La cuenca (bassin) en la que se asienta la ciudad de Valencia [...] no tiene parangón en Europa en cuanto a exhuberancia. [...]. iQue pronto se olvidan las incomodidades de los caminos y de las posadas en donde no hay ni pan para comer, en medio de la frondosidad de esta vegetación y de la indescriptible belleza física de estas gentes» ${ }^{2}$.

El 13 de mayo los dos viajeros continuaron en dirección a La Coruña, de donde zarparon el 5 de junio rumbo a Tenerife.

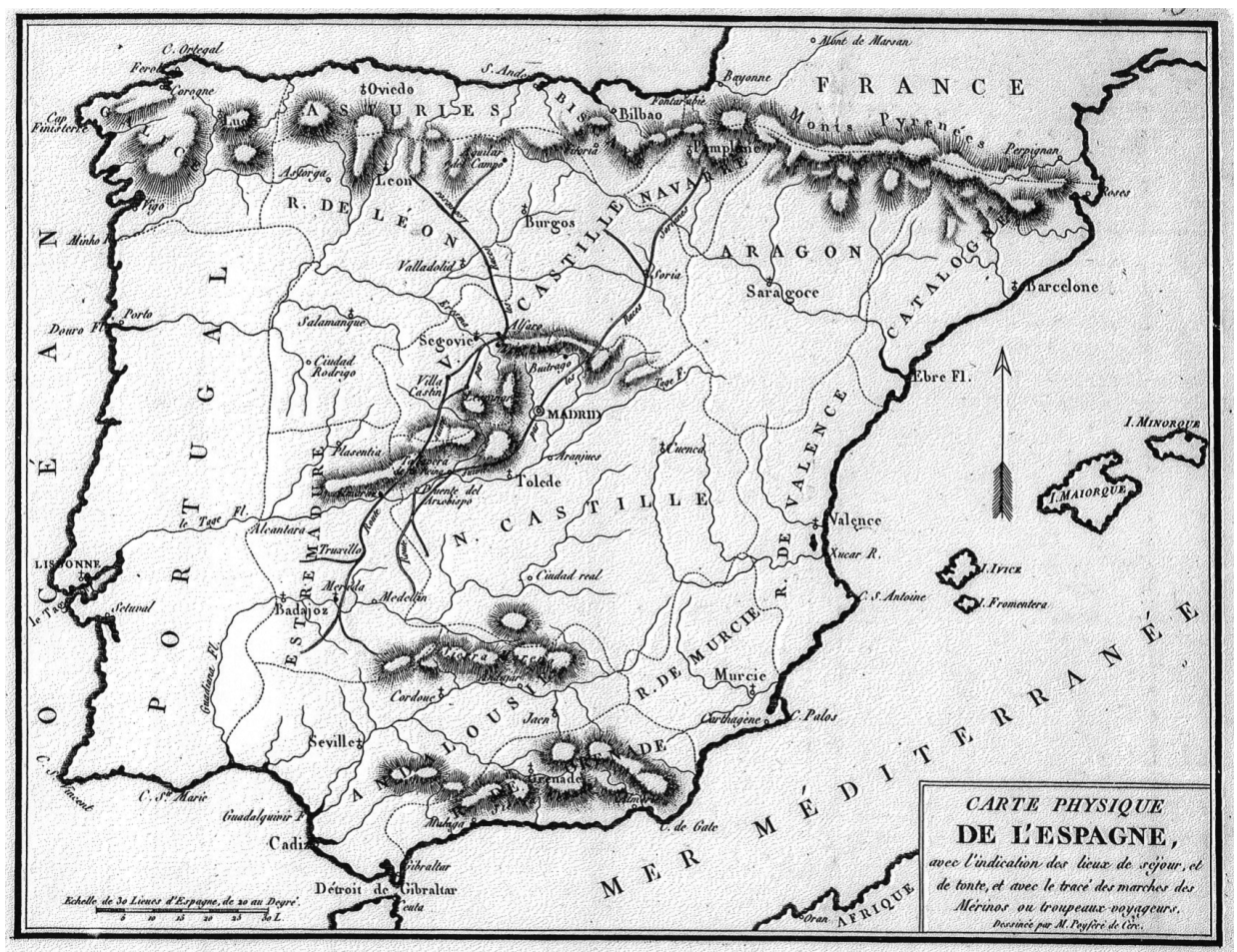

Mapa de España. Legado de Humboldt, gr. K. 5, Nr. 15, Staatsbibliothek zu Berlin-Preußischer Kulturbesitz

2 (1973), Humboldt a K.L. Willdenow, Aranjuez, 20-4-1799. En JaHN, I. y LANGE, F. (eds.), Die Jugendbriefe Alexander von Humboldts 1787-1799, Beiträge zur Alexander-vonHumboldt-Forschung, 2, Berlin, Akademie Verlag, pp. 662-663. 
Humboldt tenía desde «su primera juventud» el «deseo vehemente de hacer un viaje a países lejanos y poco visitados por europeos $»^{3}$, como comenta en la descripción del viaje publicada tras su regreso. Le ayudaron en su preparación diversos recorridos cortos por Alemania, Italia, Austria, además del aprendizaje de instrumentos y métodos de medición, así como las visitas a naturalistas y jardines botánicos. Aunque Humboldt todavía no tenía claro adónde dirigirse en su viaje al extranjero, sí manifestó el deseo generalizado «de poder observar de cerca la naturaleza salvaje, sublime y en su compleja producción» ${ }^{4}$.

Durante su estancia en París se le había ofrecido, junto a su acompañante Aimé Bonpland, la oportunidad de incorporarse al viaje alrededor del mundo de Nicolás Baudin, aplazado indefinidamente al estallar la guerra: «Defraudado dolorosamente en mis aspiraciones, al ver como en un único día se destrozaron los planes que había proyectado a lo largo de varios años de mi vida, dejé al azar prácticamente el encontrar la vía más rápida de marcharme de Europa y lanzarme a una empresa que me pudiera consolar de la pérdida que sentía», escribió Humboldt más tarde 5 .

Así pues, pretendía embarcarse en Marsella rumbo a África para recorrer a pie la cadena del Atlas y participar en la expedición francesa a Egipto. Pero también este plan fracasó, según relata: «nos vimos en la necesidad de retrasar la ejecución de nuestros propósitos y decidimos pasar el invierno en España con la esperanza de poder embarcar en Cartagena o en Cádiz, en la próxima primavera, cuando la situación política de Oriente lo permitiera» ${ }^{6}$.

Más bien fue fortuito que Humboldt viniera a España y también la casualidad determinó la ruta posterior del viaje. «Por fin, pues, rumbo a España, quizás, todavía, unos seis u ocho meses en suelo europeo. [...] Mi destino futuro demostrará si he elegido lo mejor [...]», escribió Humboldt en su diario $^{7}$. Con esta decisión se interrumpe la descripción en el diario ya publicado, pero que continuará poco después a bordo del Pizarro el día 3 de junio, antes de desembarcar en Tenerife.

El diario de España, que hasta ahora estaba sin descubrir, pertenece a este intervalo, pues no se encuentra entre los otros encuadernados de su viaje americano, sino en el legado de Humboldt.

3 Beck, H. (ed.) (1997), Die Forschungsreise in die Tropen Amerikas, Studienausgabe, II, 1-2, Darmstadt, Wissenschaftliche Buchgesellschaft, I, p. 20.
BECK (1997), I, p. 21.
BECK (1997), I, p. 23.
6 BECK (1997), I, p. 25.
7 FAAK (2000), p. 55. 
Legado y diarios: cofres donde se atesora el conocimiento

Es sabido que Humboldt no escribía diarios en el sentido estricto de la palabra, sino que durante los viajes hacía apuntes científicos que, tras su regreso, completaba con adiciones. En los diarios incluía las descripciones cronológicas de las observaciones y experimentos, así como sucesiones de medidas y cálculos que ocupan páginas enteras, extractos de bibliografía y los primeros borradores para las proyectadas publicaciones. Humboldt iba anotando todo esto en diversos cuadernos, cuya organización original no se ha conservado hasta hoy ${ }^{8}$, puesto que él mismo fue seleccionándolos para publicarlos. Sólo al final de su vida hizo encuadernar de nuevo los manuscritos del viaje americano en su forma y secuencia actuales.

Como muestran las diferentes acotaciones, en parte datadas cronológicamente, los diarios constituyeron en sí un material de trabajo a lo largo de toda su vida. Son, efectivamente, el testimonio fundamental del viaje a América, aunque también se encuentran muchos manuscritos en el legado de Humboldt de Berlín y Cracovia ${ }^{9}$. Parece que Humboldt reunió una gran cantidad de escritos a lo largo de décadas, como punto de partida para publicaciones posteriores $\mathrm{y}$, en ese sentido, es evidente que el material fue archivado, como muestra el ejemplo de las llamadas Collectaneenkästen o cajas para el Cosmos.

Todavía hoy se puede reconocer esta sistematización del contenido de su legado. Por lo visto, Humboldt no insertó en las libretas reencuadernadas las partes sueltas de los diarios que había incorporado como compilación de apuntes sobre un tema determinado, como, por ejemplo, la parte del diario de México a Veracruz $^{10}$. Es evidente que ocurrió lo mismo con su diario de España.

8 Se puede desprender una idea aproximativa de su elaborado Index général de mes Mss.; véase FAAK (2000), p. 24; LEITNER, U. (2005a), Vielschichtigkeit und Komplexität im Reisewerk Alexander von Humboldts. Bibliographischer Hintergrund. En HiN- Humboldt im Netz, VI/10, disponible en: http://www.uni-potsdam.de/u/romanistik/humboldt/hin/hin10/ leitner.htm (consultado el 24 de agosto de 2011).

9 Sobre la historia del legado, véase LEITNER, U. (2005b), Alexander von Humboldt. Von Mexiko-Stadt nach Veracruz. Tagebuch, Beiträge zur Alexander-von-Humboldt-Forschung, 25, Berlin, Akademie Verlag, pp. 7-10.

10 Legado de Humboldt 1-3, Papeles sobre la estadística de Mexiko y Cuba, Cracovia, Biblioteka Jagiellonska, véase LEITNER (2005b). 
El Diario en España y las publicaciones

En una carpeta con el rótulo De la Configuration du Sol dans la Péninsule Espagnole et du tracé de sections verticales, qui représentent du grandes étendus de pays par Alexandre de Humboldt, hallado en el legado de Humboldt en Berlín ${ }^{11}$, se encuentran los materiales recopilados por Humboldt para la publicación de «Sobre la configuración y el clima de la meseta de la Península Ibérica» en la revista Hertha ${ }^{12}$. Esto afecta también al Diario de España.

Los primeros cálculos sobre las medidas de la meseta española aparecieron en 1799, en un perfil de altitudes, en la publicación Anales de historia natural, con una introducción de Cavanilles, provista de la nota siguiente: «Las alturas desde el nivel del mar de Valencia hasta Madrid fueron tomadas por el Sr. Baron de Humboldt: las restantes [...] por D. Juan Guillermo Thalacker, Colector del Real Gabinete de Historia Natural» ${ }^{13}$. Humboldt comentó esta publicación en 1825: «Mis primeras observaciones sobre las diferencias de altura en los alrededores de Madrid fueron incluidas por Cavanilles en el primer número de los Anales de Historia Natutal, Tom. 1, pag. 86, pero mezcladas con algunas mediciones bastante inexactas de nuestro compatriota Thalacker (mineralogista bien instruido, por lo demás)» ${ }^{14}$. En realidad se trata, en el caso de Humboldt, de sólo dos datos: las altitudes de Aranjuez y Madrid. Estos resultados volvieron a imprimirse en 1808 en una obra sobre España ${ }^{15}$ «y por mí mismo está explicado con algunos comentarios que refieren al clima» ${ }^{16}$. Se trata, en estas «observaciones», de un prolijo artículo de Humboldt besitz.

11 Legado de Humboldt, gr. K. 5, Nr. 15, Staatsbibliothek zu Berlin-Preußischer Kultur-

12 Humboldt, A.v. (1825), Ueber die Gestalt und das Klima des Hochlandes in der iberischen Halbinsel, Auszug eines Schreibens an Herrn Professor Berghaus, Hertha, 40, pp. 523; Humboldt, A.v. (1869), Briefwechsel Alexander von Humboldt's mit Heinrich Berghaus aus den Jahren 1825 bis 1858, Jena, Hermann Costenoble, pp. 18-48; PUIG-SAMPER, M.A. y Reвок, S. (2007), Sentir y medir. Alexander von Humboldt en España, Madrid, Theatrum naturae, pp. 181-200.

13 Humboldt, A.v. (1799a), [Profil], Anales de historia natural, 1 (Oct.), p. 87; véase PUIG-SAMPER y REBOK (2007), p. 120.

14 Puig-Samper y ReboK (2007), p. 184.

15 Humboldt, A.v. (1808), Notice sur la configuration du sol de l'Espagne et son climat. En LABORDE, A. de (1808), Itinéraire descriptif de l'Espagne, et Tableau des différentes branches de l'administration et de l'industrie de ce royaume, vol. 1, Paris, Nicolle, pp. cxlviiclvi; véase PUIG-SAMPER y REBOK (2007), pp. 177-181.

16 Puig-Samper y ReboK (2007), p. 184. 
de varias páginas con el título de Notice sur la configuration du sol de l'Espagne et son climat, en donde describe la singular configuración de la meseta española en el contexto europeo. «L'aridité du sol dans l'intérieur des Castilles, la force de l'évaporation, le manque de rivières, et cette différence de température que nous observons entre Madrid et Naples», dos ciudades que se encuentran en el mismo grado de latitud. Humboldt no solo traza comparaciones con otras mesetas de Europa (Suiza, Francia, Alemania) sino también con las que había visitado a lo largo de su viaje americano (México). Junto a las observaciones sobre el clima, datos de altitud y especificaciones sobre el crecimiento de las plantas, añadirá también las relaciones con las particularidades geológicas.

Durante muchos años Humboldt dio por extraviado este manuscrito, es decir, la parte del diario que aquí se presenta, «y sólo en mi último viaje a Alemania ha vuelto a mis manos» ${ }^{17}$, probablemente en 1823 . Quizás ese sea el motivo por el que la meseta española no jugó ningún papel en su Essay gégnostique $^{18}$, publicado en 1822-23.

El 16 de agosto de 1825 Humboldt informaba a Heinrich Berghaus, editor de la revista Hertha (Cotta, Stuttgart) sobre una nueva publicación. «[...] Emprendo una memoria sobre la configuración y el clima de la meseta española. La redactaré en forma de carta dirigida a usted, por la brevedad, pero la enviaré directamente a Stuttgart [...], puesto que a Cotta le urge que le envíe algo escrito para el próximo número de Hertha» ${ }^{19}$. El artículo, firmado en París el 6 de septiembre de 1825, fue enviado a Cotta el 7 de septiembre ${ }^{20}$.

Como anexo al texto aparecido en la revista Hertha, en el cuarto volumen del año, se halla el perfil de altitudes de la meseta española que Humboldt acababa de dibujar en París en 1823, y que había publicado en 1825 en el Atlas $^{21}$ perteneciente a la obra del viaje americano. Esta tabla consta de dos perfiles: el primero se despliega desde Valencia hasta La Coruña a través de Madrid, es decir, se corresponde con el trayecto de su viaje y de sus compro-

17 Puig-SAMPER y ReBOK (2007), pp. 184-185.

18 Humboldt, A.v. (1823), Geognostischer Versuch über die Lagerung der Gebirgsarten in beiden Erdhälften, Straßburg, Levrault.

19 Humboldt (1869), p. 17.

20 Carta de Humboldt a Cotta, 7-9-1825, Archivo Cotta, Schiller Nationalmuseum Deutsches Literaturarchiv (Stiftung der Stuttgarter Zeitung), Marbach.

21 Humboldt, A.v. (1814-1838), Atlas géographique et physique des régions équinoxiales du Nouveau Continent, fondé sur des observations astronomiques, des mesures trigonométriques et des nivellements barométriques, Paris, Gide, Pl. III; véase PUIG-SAMPER y REBOK (2007), pp. 186-187. 
baciones; el segundo, desde Sierra Nevada hasta los Pirineos, según las medidas de Thalacker y otros.

Sobre el contenido del diario de España

El presente diario consta de varias partes. Dos textos más extensos contienen la primera descripción geológica del recorrido del itinerario desde Valencia a Madrid y desde Madrid a La Coruña. Como se puede inferir de la edición, estas partes del diario fueron, en parte, reproducidas en la publicación de 1825 de Hertha. Se han recogido los correspondientes pasajes en las notas finales, para su comparación. Evidentemente existe una diferencia básica, pues los datos de altitud difieren, en parte, de forma considerable. Por este motivo no aparecen en las notas a pie de página de los textos que se comparan.

En el diario hay dos tipos de datos de altitud. Por una parte, los que aparecen en el texto continuo, y por otra, los que se indican en la primera página, en una lista con los datos fechados en mayo de 1799. Por añadidura, Humboldt expresó durante la segunda parte del viaje, en el recorrido de Madrid a La Coruña, que todavía esperaba desde Madrid los niveles barométricos. Tuvo finalmente que obtenerlos, puesto que en 1825 se expresa de la siguiente manera: «Los altitudes barométricas comparadas las obtuve, en parte, en Valencia, a través del señor Valenzuela; en parte en Madrid, por la bondad del señor Chaix ${ }^{22}$. Mientras, para la publicación «he continuado ininterrumpidamente recogiendo, por correspondencia, todas las informaciones que tienen relación con las diferencias de altitud y el clima de la península española» ${ }^{23}$.

El instrumento de medición de Humboldt, el «barómetro era uno de cubeta según el modelo de Ramsden » ${ }^{24}$, con el que se determinaba la presión del aire en líneas, la unidad de medida del barómetro. Con este barómetro calculó la altitud en toesas, según diversas fórmulas (las de Deluc, Laplace y Trembley). Según la fórmula utilizada, los resultados finales resultan diferentes. Puesto que con la medida barométrica se percibía una diferencia de altitud entre dos lugares, resultó imprescindible determinar una altitud concreta y exacta como punto de partida. Humboldt escribió sobre esto: «En todos los lugares donde he pernoctado he hecho cuidadosamente la observación por la mañana y al anochecer y $[\ldots]$ he puesto a un punto en relación con el otro. Para mí lo mas

22 PUIG-SAMPER y REBOK (2007), p. 185.

23 Puig-SAMPer y ReBOK (2007), p. 185.

24 Puig-SAMPer y ReBoK (2007), p. 185. 
importante era dilucidar la altitud de Madrid, como punto central, mediante la comparación de muchos datos, independientes entre sí, para luego calcular, en ambas Castillas y Astorga, las localidades debajo y sobre el horizonte de Madrid» $»^{25}$. Para este método relativo, el perfil de altitudes empleado por Humboldt resulta manifiestamente un medio adecuado de percepción.

A lo largo de un intercambio epistolar de varios decenios con el Director interino del Depósito Hidrográfico de Madrid, Felipe Bauzá, Humboldt recibió otras medidas barométricas, especialmente de esta ciudad, que comparó con las suyas propias ${ }^{26}$. Para la edición de 1825 , es decir, para el perfil publicado en 1823, Humboldt calculó de nuevo «las alturas barométricas de este manuscrito [=su diario]» ${ }^{27}$, de lo que se desprenden las diferencias entre los datos de altitud en el diario y en la publicación.

En el presente diario, entre las dos partes del manuscrito que describen el viaje, aparece, en la página siete, una enumeración de plantas que florecían en Valencia, en enero de 1799, y hace alusión al «libro de Bonpland». El acompañante de Humboldt tenía autoridad para el análisis botánico del viaje y con este fin manejaba un diario aparte, en el que se indican las plantas recolectadas, cronológicamente numeradas, con la descripción botánica, el lugar del hallazgo y los datos de altitudes ${ }^{28}$.

Asimismo en la página siete Humboldt aclara el método que ha utilizado para medir las distancias. Para ello caminaba regularmente 120 pasos por minuto, que se corresponden con 270 pies de París $(=87,67 \mathrm{~m})$. Así, se podía concluir el tiempo empleado con pasos rápidos y uniformes en un recorrido y la distancia andada. Humboldt utilizó este método para determinar «bases grandes de 300 toesas» $(=584,52 \mathrm{~m})$, que se utilizaban para la triangulación. Con ayuda de estas bases y con la medición de los ángulos que Humboldt realizaba con el sextante, por medio de relaciones trigonométricas, se podían determinar distancias largas, intervalos de puntos, así como la exactitud de las altitudes. Para la precisión de este método resultó determinante el establecimiento correcto de la base. Humboldt le dedicó una reflexión en forma de nota en el diario.

25 PUIG-SAMPER y REBOK (2007), p. 185.

26 Véase LeITNER, U. (2005c), Los diarios de Alexander von Humboldt: un mosaico de su conocimiento científico. En ReboK, S. y Cuesta Domingo, M. (eds.), Alexander von Humboldt. Estancia en España y viaje americano, Madrid, Real Sociedad Geográfica, pp. 163-176.

27 PUIG-SAMPER y REBOK (2007), p. 185.

28 Este Journal botanique, hasta ahora no publicado, se encuentra hoy en París, Muséum national. Véase LACK, H.W. (2004), The botanical field notes prepared by Humboldt and Bonpland in tropical America, Taxon, 53 (2), pp. 501-510. 
En el presente diario se hace también alusión a otra nota históricocientífica interesante: Humboldt visitó plantaciones de nitrato en Quintanar de la Orden. En el diario (p. 5) se halla una referencia a su artículo publicado en Annales de chimie. Humboldt había concluido poco antes su libro Versuche über die chemische Zerlegung des Luftkreises (Ensayos sobre la descomposición química de la circulación del aire), en Marsella, y trabajos diversos que, en parte, como este artículo, ya habían sido publicados en otros lugares.

El libro contenía los resultados de investigación sobre los estudios químicos y barométricos de análisis de la atmósfera, a partir del año 1797. El salitre o nitrato se forma por la descomposición debida a la influencia del aire, y se utilizaba para la elaboración de pólvora para munición y pólvora de minas, para la fabricación de cristal y también como medicamento. En algunas regiones abunda de forma natural y, en otras, se provoca su formación de manera artificial, al acumular cantidades de ciertas sustancias concretas como escombros, basuras, cenizas, etc. Humboldt ya había descrito en su publicación semejantes «yacimientos artificiales de salitre». «En Alemania se erigen muros de barro en los campos, que se disponen paralelamente y sobre los que el salitre se va apilando». Humboldt asegura que «es posible que una parte de la atmósfera se pueda transformar en ácido nítrico, bajo el influjo de la electricidad». Aunque esta teoría contradice el hecho de que, cómo había observado, «el salitre genera mayor volumen sobre arcilla y tierra caliza que sobre cuarzo [...], tiene que existir una relación exacta entre la formación del salitre y la naturaleza de las sustancias sobre las que se descompone» ${ }^{29}$.

Humboldt había descrito los experimentos en su publicación, llevados a cabo junto con el químico Nicolás Louis Vauquelin, en el laboratorio de París. Con todo ello había intentado desarrollar una «ley química general» que esencialmente se corresponde con un análisis cuantitativo.

Además, en la página 8 se encuentra un pasaje sobre el «clima» $»^{30}$, en el cual Humboldt declara su admiración. A pesar del calor en Valencia $\left(18^{\circ}\right.$ a la

29 Humboldt, A.v. (1799b), Mémoire sur l'absorption de l'oxigène par les terres simples, et son influence dans la culture du sol, Annales de chimie, 29 (an VII, 2), pp. 125-160. Citado de la versión alemana: Ueber die Verbindung der Erden mit Sauerstoff oder: über die Absorption des Sauerstoffs durch die einfachen Erden und dessen Einfluss auf die Kultur des Bodens. En Humboldt, A.v., Versuche über die chemische Zerlegung des Luftkreises, Braunschweig, Vieweg, pp. 145-147.

30 Sobre la contribución de Humboldt al desarrollo de la climatología, véase BERNHARDT, K.-H. (2003), Alexander von Humboldts Beitrag zu Entwicklung und Institutionalisierung von Meteorologie und Klimatologie im 19. Jahrhundert. En HAMEL, J., KNOBLOCH, E. y PIEPER, H. (eds.), Alexander von Humboldt in Berlin. Sein Einfluß auf die Entwicklung der 
sombra), los árboles y arbustos no tienen hojas ni renuevos, justo lo contrario que en Berlín, en donde el calor permite que los árboles germinen en el plazo de un periodo de tiempo muy breve. Después especuló sobre las causas: bien una «irritabilidad de las plantas», acaso almacenada durante el sueño hibernal, o bien el oxígeno disuelto en la nieve.

Humboldt había reunido datos sobre las temperaturas medias de Madrid, Karlsruhe, Marsella, París y Palermo en la publicación de 1825, pero no proceden de su diario porque fue posteriormente recibiéndolas de otros por correspondencia, como, por ejemplo, los datos sobre Madrid de Felipe Bauzá. Precisamente en la publicación anterior de 1808 aparece también un cuadro con datos comparativos sobre las temperaturas medias en Madrid y en Roma. Los últimos aportes proceden de su hermano Guillermo. Al comparar las temperaturas medias anuales, su evolución en el curso de un año y los grados de latitud, pudo hallar las causas de por qué determinadas plantas, como los cítricos, no prosperan en la meseta aunque si abundan en otros lugares que tienen la misma latitud ${ }^{31}$.

En el diario, la medida de la electricidad de la luz, realizada con un electrómetro atmosférico ${ }^{32}$ (pág. 10), pertenece también al apartado sobre las observaciones del clima cuando se produjo una tormenta el día 28 de mayo en La Coruña. Se conocía la relación entre el clima y la electricidad del aire, concretamente la transformación que se produce al aproximarse una tormenta.

Wissenschaften. Beiträge zu einem Symposium, Algorismus. Studien zur Geschichte der Mathematik und der Naturwissenschaften, 41, Augsburg, Erwin Rauner Verlag, pp. 195-221; KÖRBER H.-J. (1959), Über Alexander von Humboldts Arbeiten zur Meteorologie und Klimatologie. En Alexander von Humboldt. Gedenkschrift zur 100. Wiederkehr seines Todestages, Berlin, Akademie-Verlag, pp. 287-325 y PUIG-SAMPER y REBOK (2007), pp. 75-76.

31 «La considerable altitud del suelo en la Península Ibérica modifica el clima de una manera peculiar con un frío seco invernal. [...] El mes más frío en la meseta de España, situada tres grados de latitud mas al sur que Marsella, es de $2^{\circ}, 7$ más fró que en esta, y en cambio el mes de julio es casi $6^{\circ}$ más cálido por la insolación térmica en la meseta desarbolada. [...] Mientras que las mesetas ibéricas interiores de más de 2000 pies de altitud tienen un verdadero clima continental, con una temperatura media de $15^{\circ}$, reina en las costas, en el crudo invierno y en el caluroso verano, en la esplendida región adornada por naranjos y palmeras datileras, que circunda las mesetas, una temperatura media de $17^{\circ}$. Citrus prospera en importante cultivo al aire libre sin protección allí, donde esa temperatura media anual de $16^{\circ}-17^{\circ}$ y la temperatura en invierno se mantiene sobre $9^{\circ}$ o $10^{\circ} »$ (PUIG-SAMPER y REBOK (2007), pp. 198-200).

32 El electrómetro fue perfeccionado en el siglo XVIII. Sobre la historia y la descripción del electrómetro, véase BRAND, F. (2002), Alexander von Humboldts physikalische Meßinstrumente und Meßmethoden, Berliner Manuskripte zur Alexander-von-Humboldt-Forschung, 18, Berlin, Alexander-von-Humboldt-Forschungsstelle. 
Humboldt incluye en la lista de instrumentos que llevaba en el viaje «dos electrómetros, el de Bennet y de Saussure, con oro laminado y bolitas de corcho, con conductores de cuatro pies de altura, para, según el método de Volta, concentrar la electricidad atmosférica por medio de una sustancia combustible que exhala humo» ${ }^{33}$. El principio era fácil: en un recipiente de cristal se encuentran las bolitas de pulpa de saúco móviles en dos pajas flotantes. La fuerza de la repulsión se considera como la medida de la electricidad. La propuesta de encerrar el electrómetro en un tubo de cristal procede de Tiberius Cavallo. Nicolás Théodore de Saussure la mejoró utilizando bolas de pulpa de saúco en vez de bolas de corcho. Abraham Bennet empleó dos cintas de pan de oro que se fijaban en paralelo y próximas una a la otra. En las mayores alturas se podía medir con un conductor. Volta aconsejó una variante que Bennet ya había propuesto para una mejor medición: «atraer» la electricidad atmosférica a través de una mecha encendida de azufre $u$ otro elemento. Después también se utilizó como método de observación el humo de yescas ardiendo, y este debió ser, evidentemente, el método que Humboldt empleó en La Coruña. En su viaje posterior realizó mediciones con diferentes métodos, por lo que, en cada caso, anotaba en el diario: «electrómetro de Volta», armé de fumée, o también non armé de fumée.

En un capítulo de la Relation historique, redactado alrededor de 1818 y dedicado al clima de los trópicos, resumía sus observaciones teóricas recogidas a lo largo de su viaje americano, donde menciona también las mediciones europeas en la comparación de datos. Para citar un ejemplo escribe que la electricidad del aire asciende «tres veces más de lo que yo solía observar en Europa con el mismo instrumento y con tiempo despejado» ${ }^{34}$.

En la descripción del itinerario predominan considerablemente los apuntes de rasgo geognóstico, como la especificación de la clase de roca y la dirección del desplazamiento y del buzamiento. Como ya se ha comentado reiteradas veces ${ }^{35}$, la verificación de la homogeneidad en el buzamiento y en la dirección y el paralelismo de los estratos en los diferentes continentes eran el

33 BECK (1997), I, p. 36.

34 BECK (1997), I, p. 166.

35 Beck, H. (1959), Alexander von Humboldt. Vol. 1: Von der Bildungsreise zur Forschungsreise 1769-1804, Wiesbaden; BECK, H. (1961), Alexander von Humboldt. Vol. 2: Vom Reisewerk zum "Kosmos" 1804-1859, Wiesbaden, I, pp. 169-173 у BECK, H. (1957), Das literarische Testament Alexander von Humboldts 1799, Forschungen und Fortschritte, 31 (3), pp. 65-70; HöLDER, H. (1994), Ansätze großtektonischer Theorien des 20. Jahrhunderts bei Alexander von Humboldt. En Studia Fribergensia, Beiträge zur Alexander-von-HumboldtForschung, 18, Berlin, Akademie Verlag, pp. 63-73. 
principal objetivo de Humboldt al emprender el viaje científico. Con ello proyectaba descubrir leyes sobre la construcción del cuerpo terráqueo, como le comunicó por carta a Karl Maria Ehrenbert, Barón von Moll el día anterior a la partida desde La Coruña: «Sigo trabajando como siempre sin interrupción en mi obra sobre la construcción del cuerpo terráqueo [...]. Ya he visto la mayor parte de Europa y me asombro cada vez más de la maravillosa sencillez de la construcción. Se pueden observar de tres a cuatro estratos desde Moscú hasta Cádiz, desde Suecia hasta Akruim en el Egipto central. Incluso he visto aparecer nuestra grauvaca en el Reino de León, igual que en el Harz, [...] debajo de los antiguas vetas calizas (piedra caliza o zechstein de los Alpes) ${ }^{36}$.

Efectivamente, en el presente diario se encuentran descripciones de estratos de muchos lugares, la mayoría con los correspondientes datos de la dirección del desplazamiento, así como comparaciones con similares estratificaciones en regiones ya visitadas anteriormente. El material reunido debía servir para hallar un parentesco en la formación de cordilleras, muy alejadas unas de otras, para de ahí deducir una ley general respecto a su génesis. Humboldt explicó más exactamente esta idea en los apuntes geológicos originales de Sudamérica, que el 15 de noviembre de 1800 enviara a Delamétherie, editor del Journal de Physique, en donde este artículo apareció después. En la introducción escribió que «en trazos gruesos» había descrito «la forja de la Tierra, la pendiente del país, la dirección y la inclinación de la formación montañosa, su edad relativa, su similitud con las formaciones en Europa» para iluminar mejor el conocimiento de «la formación de la Tierra [...]. Los fatigosos viajes que he realizado en Europa en los últimos ocho años tenían justamente este objetivo, y si tengo la dicha de regresar a Europa [...], confío en poder atreverme a dibujar un esbozo de la forja de la Tierra. Tiempo habrá de demostrar lo que he mantenido durante tantos años: que la dirección y la inclinación, el desplazamiento y el buzamiento de las primitivas capas terrenas, el ángulo que se forma con el meridiano del lugar y con el eje terrestre, son independientes de la dirección y de la pendiente de las montañas, y que se rigen por leyes que evidencian un paralelismo general que sólo puede estar fundamentado en la gravitación y en la transformación espasmódica de la Tierra. Quedará corroborada [...], que hay una identidad en los estratos de las formaciones, de lo cual se deberá deducir que los mismos depósitos se han originado en toda la superficie de la Tierra en el mismo periodo de tiempo» ${ }^{37}$. De ahí que se pueda confirmar el concep-

36 JAHN y LANGE (1973), p. 682.

37 HumboldT, A.v. (1801), Esquisse d'un tableau géologique de l'Amérique méridional, J. phys., chim., hist. nat. et arts, 53, p. 61. Citado de la versión alemana: (1802), Skizze einer 
to, formado a partir de la similitud de la forma de los continentes, de que éstos fueron separados por la fuerza del agua ${ }^{38}$.

Humboldt no llegó a publicar una obra sobre la construcción del cuerpo terrestre, posiblemente porque no pudo demostrar esta correspondencia con las observaciones efectuadas durante el viaje, si bien todavía expresó en su publicación de 1825 los datos recogidos en el diario sobre la dirección de los estratos, precisamente cuando se había distanciado de una ley general del paralelismo en el Essai géognostique ${ }^{39}$, publicado en 1823. No obstante, seguía interesado ${ }^{40}$, como antes, en trazar analogías al presentar, en la publicación de 1825 (por ejemplo, las notas finales ii y xli del diario), la aceptación de paralelismos, ya constatados en el diario, en la disposición de los estratos entre España y la región anteriormente visitada. Por el contrario, se echa en falta, en la publicación de 1825, una observación sobre la dirección significativa en España, en la Provenza y en Col de Balaguer (nota final xviii).

Entre tanto, Humboldt también había aceptado la teoría del levantamiento, sostenida por Leopold von Buch, como señalan dos notas ${ }^{41}$ en la publicación de 1825 que, por otra parte, no se hallan en los contenidos casi idénticos del texto del diario, un ejemplo ilustrativo sobre el desarrollo de los principios teóricos de Humboldt entre 1799 y 1825.

Puesto que el presente diario de España es un manuscrito sobre el inicio de su viaje científico, ha sido interesante poder constatar que también éste se corresponde con las características del resto de sus diarios en el estilo típico de redacción como un work in progress. Es decir, una combinación de la narración y descripción del viaje, sucesiones de medidas expresadas en tablas, primeras reflexiones conceptuales sobre problemas científicos y observaciones, posteriormente anotadas al margen, con las coordenadas geográficas coregidas por cálculos de medidas.

Geologischen Schilderung des südlichen Amerika, Allgemeine Geographische Ephemeriden, 9 (St. 4 vom April), pp. 310-329; (St. 5 vom Mai), pp. 312-313.

38 HumboldT (1801), 317.

39 Humboldt (1823), 61.

40 Cómo Hölder ya mostró para el Essai géognostique, véase HöLDER (1994), p. 64.

41 «En este último país [Francia] he observado frecuentemente, [...] que, incluso allí donde la llanura se interrumpe por barrancos o por pequeñas cadenas montañosas, su elevación, a lo largo de 50 ó 60 millas, como llanura alcanza siempre el máximo. ¿Indica este fenómeno una igualdad de las fuerzas interiores elevadoras?» (PUIG-SAMPER y REBOK (2007), p. 190). «La caída de los estratos debilitados parece indicar también aquí las potentes elevaciones, cuya conexión causal con otros fenómenos geognósticos han desarrollado Friedrich Hoffmann y Leopold van Buch» (PUIG-SAMPER y REBOK (2007), p. 191). 


\section{EDICIÓN DEL MANUSCRITO}

Principios de edición

El texto completo reproduce lo mejor posible y de forma íntegra el correspondiente manuscrito. Las lecturas inciertas aparecen entre paréntesis. Se han conservado las peculiaridades del estilo de redacción de Humboldt.

Se indican por orden numérico los «saltos de página» del original.

Las «notas al final del texto» contienen los comentarios de la editora. Aquí el lector puede encontrar los textos paralelos de la publicación, que Humboldt publicó en 1825 en el diario Hertha, citado de la traducción en PUIG-SAMPER y REBOK (2007).

En las «notas a pie de página» se encuentran los elementos críticos, a los que pertenecen:

- Las observaciones al margen del propio Humboldt. Las referencias a pié de página están situadas en el texto, al que completan. Las observaciones al margen que no podían agregarse de forma clara aparecen en forma de referencia a pié de página del diario.

- Tachaduras (la palabra anterior y posterior a la tachada se repite en la nota del pié de página), por ejemplo:

Castilla la Vieja. León ${ }^{1}$

${ }^{1}$ Castilla la Vieja | Castilla la Nueva tachada | León $H$

- Apéndices, por ejemplo:

Días de verano ${ }^{2}$

${ }^{2}$ verano añadido por Humboldt

- Se reproducen las variantes con (1) (2)

Se han completado implícitamente en la trascripción al alemán los acentos, diéresis y demás signos de puntuación absolutamente necesarios para la comprensión del manuscrito.

Se han completado las abreviaturas de Humboldt con corchetes, por ejemplo mer[idional], E[rica].

EL DIARIO

/1/ Desde Valencia hasta Madrid ${ }^{1}$

1 H. en el margen:

Scripsi en el Reino de León, en Astorga y Villafranca, mayo 1799.

Barómetro (añadir 37 líneas*) 
Enormes vergeles, incluso una hora después de haber dejado Valencia. Cultivos únicos. Poco después, arados en vez de labor manual, sembrados en vez de huertasi.

Gradualmente terrazas más desnudas y más pequeñas. Finalmente, roca caliza sin arbolado // La misma formación que aparece en Tarragona, Oropesa, La Mancha y en el Reino de León y que se sitúa bajo el conglomerado de nagelflú $o^{\mathrm{ii}}$, como en Balaguer y en el Montserrat. // Por la mañana se ve el promontorio de Cullera a la izquierda. Se eleva enérgico como las masas rocosas de Marsella Veine entre Toulon y Marsella, también con esa misma formación y similar configuración. Alcudia, un pueblo en un emplazamiento precioso. En las proximidades Schinus mollis ${ }^{2}$, al aire libre, plantado en torno a un lugar de peregrinación ${ }^{i i i}$. Alginet ${ }^{3}$ a 42 toesas $^{\text {iv }}$ sobre el mar iy a partir de ahora siempre sobre el mar! Nos aproximamos a la sierra que se extiende de

$\begin{array}{ll}\text { Valencia } & 301,8 \\ \text { Alginet } & 298, \\ \text { Sierra Santa Ana } & 297,4 \\ \text { mojón número 53 } & 294,5 \\ \text { Venta de Morente } & 291,2 \\ \text { Puerto de Almansa } & 281,2 \\ \text { mojón número 49 } & 279,2 \\ \text { Almansa } & 277,4 \\ \text { Bonete } & 271,2 \\ \text { mojón número 42 } & 270 \\ \text { Venta del Rincón } & 272,2 \\ \text { Albacete } & 279,6 \\ \text { La Roda } & 278,4 \\ \text { Minaya } & 277,5 \\ \text { El Provencio } & 278,8 \\ \text { El Pedernoso } & 278,5 \\ \text { Quintanar de la Orden } & 279 \\ \text { El Corral de Almaguer } & 278,4 \\ \text { Ocaña } & 276,2 \\ \text { Aranjuez } & 285 \\ \text { Valdemoro } & 281,2 \\ \text { Madrid } & 280 *\end{array}$

Las alturas se calculan con más exactitud desde estas posiciones [del barómetro].

$(1) * j$ 'ajoute 34 li pour $\{$ ilegible $\}$ pour Madrid la haut[eur] \{ilegible $\}(2) *$ en ajoutant à Valence et Madrid $37^{\mathrm{li}}$ on trouve Madrid 290 toesas pour trouver 311 toesas je mets | 300,0 tachado | Madrid 280 $0^{\text {li, }}$ la valeur 302,5

2 mollis | salvaje tachado | en $H$.

3 plantada. | Valencia bar. 301,1. tachado | Alginet $H$. 
Este a Oeste en la parte sur del Reino de Valenciav . Cerca de ella, se desvía el río Júcar, de una anchura de 80 pies, por cuyo motivo se construye un puente enormemente valioso. Sierra de Santa Ana, de caliza, hasta 54 toesas de altitud. Después una formación de greda sobre aquella y, en ésta, un extenso estrato de yeso que supera las 3.000 toesas $^{\mathrm{vi}}$. Yeso antiguo ${ }^{4}, / 2 /$ granuloso, $^{2}$ foliado, infiltrado de greda, la misma que contiene sal gema en Villarrubio, en La Manchavii, idéntica a la de Alande en Marsella y la de Aarau en Suiza. Máxima altitud, 78 toesas, precisamente la altitud también de Venta del Col, en Sumacárcel. En ésta, otra vez roca caliza. El mismo yeso está al descubierto, incierto si sobre él por otra parte yacía piedra caliza. Yeso irregular, la mayor parte a 9 horas al sur. La sierra de roca caliza siempre en ascenso. Un valle cultivado entre dos sierras calcáreas, dentelleadas, pobladas de bosques viii. 700 toesas antes del mojón 53, la superficie se eleva en pendiente ascendente a 105 toesas de altitud. El punto más alto de esta planicie es de 165 toesas (= 990 pies de altura), comprendido ${ }^{5}$ entre la sierra de Santa Ana y el puerto de Almansa. La cadena de colinas se desarrolla junto al camino 4-5 horas, a lo largo de una altitud de 240 toesas ${ }^{\text {ix. }}$. Venta de Morente (150 toesas). Después del mojón 51 aparece un fenómeno muy notable. La cadena de colinas de 4 horas se despliega siempre hacia la parte occidental. Los estratos de piedra caliza se extienden 4,2 horas con $30^{\circ}$ de descenso hacia el sureste. Aquí siguen cuatro hendiduras, una tras otra, que caen cortadas, en vertical, desde la colina. La parte de roca que falta está desprendida y se encuentra en el fondo. En los bancos que descienden a $60-70^{\circ}$ se reconoce visiblemente el orden anterior ${ }^{\mathrm{x}}$.

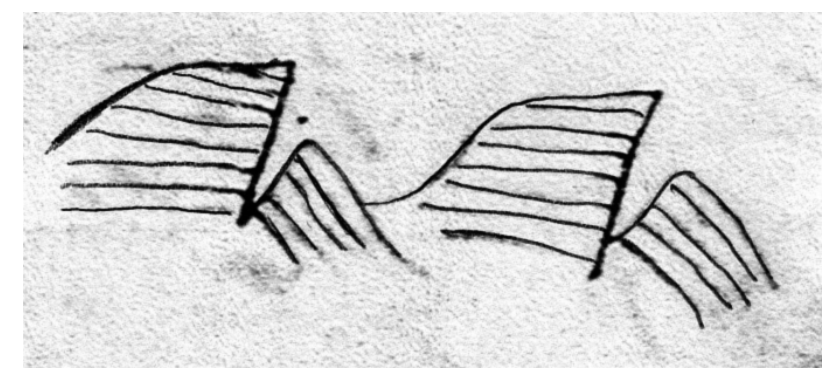

4 H. en el margen: 460.

$$
\frac{340 .}{800}
$$

5 esta planicie está comprendida entre la sierra de Santa Ana y el Puerto de Almansa, añadido por $H$. 
13/ Después un monte escarpado calcáreo, el Puerto de Almansa, cuya cumbre alcanza 300 toesas. A partir de allí, una superficie, una meseta alta rodeada en todas direcciones de cadenas de peñascos. Posiblemente un fondo marino, un zócalo antiguo en suave ascenso ${ }^{\mathrm{xi}}$. En el mojón 49 aparece a 332 toesas la Heide Erica vagans Erica mediterránea con algunos sembrados. Cerca de la ciudad de Almansa, las ruinas del viejo castilloxii se erigen como una isla en la inmensa planicie a escasas 15 toesas sobre la roca. La ciudad, 364 toesas. Tras Almansa, el fondo marino, el zócalo encerrado en cierto modo por una preciosa pendiente delineada hacia el norte y que se precipita en vertical, de 4 horas, interrumpida como un dique en la parte oriental y la occidental.

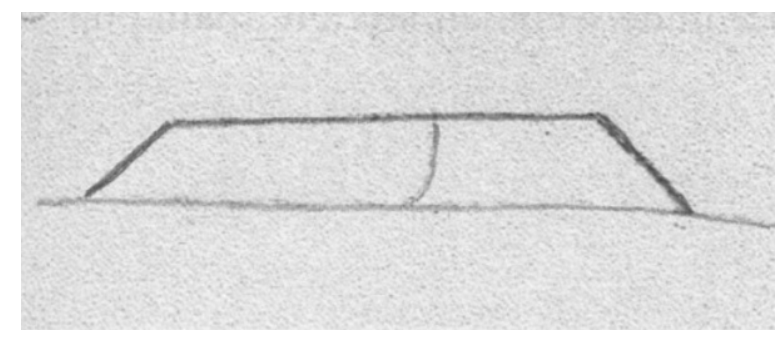

La superficie calcárea de la meseta sigue siempre en ascenso hasta Bonete (480 toesas). Ningún árbol; monotonía interminable. En el mojón 42 se encuentra el punto más elevado de la altiplanicie (468 toesas) y la cadena de colinas de al lado, aproximadamente de 550 toesas (=3.300 pies de altura). Allí sobre la piedra caliza se extiende una formación de piedra arenisca no muy amplia, que parece ser distinta de la formación de conglomerados de nagelflúo. La arenisca es muy similar al pórfido, muchos cantos rodados de cuarzo con cemento en forma de guijarros y dentro nódulos ferruginosos ${ }^{x i i i}$ color marrón. A partir de ahí empieza a descender gradualmente la llanura. Esta pendiente, detrás de la Venta del Rincón (432 toesas), es ciertamente la superficie más rica en cereales de La Mancha. En Albacete (315 toesas), constante formación calcárea del Jurásico. La Roda, /4/ (331 toesas). Minaya $(344)^{6 x i v}$. Tras Minaya, la formación calcárea se hace muy porosa, con burbujas, la misma transformación de roca caliza jurásica que aparece en Streitberg, en Franconia, cerca de Muggendorf ${ }^{\mathrm{fv}}$. El Pedernoso, en El Provencio (331

6 (1) 298 toesas Minaya 331 toesas (2) La Roda 331 toesas Minaya $344 H$. 
toesas) ${ }^{7}$. Muchas terrazas y en ellas gran cantidad de cantos con conchas fosilizadas en transición a calcedonia, muy bonitas, en parte con formas arracimadas y alargadas, arriñonadas, muy bonitas. Después de juzgar muchos fragmentos, seguramente piedra caliza. Similar a la formación de Vallecas en Madrid y a la de París, de piedra molarxvi. Junto a El Toboso se encuentra piedra arenisca sobre roca caliza, muy cuarzosa, de grano fino, compuesta de granos de cuarzo muy redondos. Son estratos como los que se ven en Aranjuez que alternan ${ }^{8 x v i i}$ con conglomerado de nagelflúo, de grano grueso como los de Montserrat y Marsella. Aquí la arenisca que es muy espesa, 10 horas con $30^{\circ}$ septentrional, no parece muy extendida. Piedra caliza en todo el norte de Murcia y en La Mancha, a 8 horas, con inclinación unas veces al norte otras al sur, con $8-9^{\circ}$, es decir la misma extensión principal que en El Provencio y en Col de Balaguerxviii.

Quintanar de la Orden (345 toesas). Allí yacimientos artificiales de salitre en montones cónicos. El suelo arcilloso cubierto completamente por salitre. Aquí las mismas proporciones que en Cujavien ${ }^{\mathrm{xix}}$ y Magdeburgo. Una superficie fértil, rica en cereales y gran humedad en muchas hondonadas. La misma causa que estimula el crecimiento de las plantas, mezcla de tierra que la atmósfera descompone fácilmente y que también produce salitre. Mémoire sur l'absorption de l'oxygène par les terres ${ }^{\mathrm{xx}}$. La mayor producción de salitre se da en Alcázar de San Juan en donde también hay molinos de pólvora a 4 leguas de Quintanar. El Corral de Almaguer (354 toesas). Ocaña (384 toesas). Media hora después de pasar por Ocaña se entra en el muy ancho valle del Tajo, excavado por un río enorme ${ }^{9}$ del que sólo queda un cauce menor ${ }^{x \times i}$. En el valle se elevan un número de pequeñas colinas calcáreas, picos truncados de apenas 20 toesas de altitud similares a los de Jena, que se elevan erguidos $^{x x i i}$ en el centro en forma de isla y de fortaleza. En Aranjuez todas las colinas de yeso foliado, a menudo de 50 brazas de espesor, atravesadas intensamente con arcilla y separadas por estratos de roca caliza, es decir, filones diversos de yeso alrededor de los filones de cal. Dentro, muchas cavidades ${ }^{\text {xxiii. }}$

Viaje a Villarrubio. Más arriba de las ruinas de ${ }^{10}$ Orecas, se encuentra una ermita sobre el arco de yeso, en el que hay un filón de sal gema en la arcilla

\footnotetext{
7331 toesas añadido por $H$.

8 H. al margen: Los estratos toscamente granulados contienen más cal, los de grano fino solamente cantos de cuarzo. La arenisca cuarzosa junto a El Toboso y Ocaña, debido a la proximidad del granito de Toledo, proporciona la arena de granito.

9 Corriente $\mid$ una tachado $\mid H$.

10 de las ruinas de añadido por $H$.
} 
salina. Las mismas condiciones que en Hallein. La montaña de Villarrubio, quizás única en su especie, debido a la enorme abundancia de profundas grietas demasiado abiertas de 2 brazas y 2 pulgadas en las que la roca ha reventado ${ }^{11 x x i v}$. Muy probablemente consecuencia de las precipitaciones que corroen la sal gema ${ }^{\mathrm{xxv}}$. La pasarela, muy peligrosa, puesto que a menudo construye puentes naturales de apenas 15 pulgadas de ancho sobre los precipicios /6/ y barrancos. La mina de sal gema yace abandonada desde hace un año a pesar de que la nueva construcción costó mucho dinero porque se descubrió una mina de sal muy rica en la otra parte del Tajo, en la orilla derecha. Aranjuez, en el valle, 238 toesas. Yeso en la cal hasta Madrid. En los valles se extiende conglomerado de nagelflúo de grano grueso y grano fino, calcáreo, aunque cerca de Toledo mas bien cuarzoso sobre roca caliza ${ }^{\mathrm{xxv}}$. Probablemente este conglomerado de nagelflúo se extiende también sobre el yeso, como en Montmartre, pero todavía no es notorio y el yeso se encuentra extendido al descubierto por todas partes.

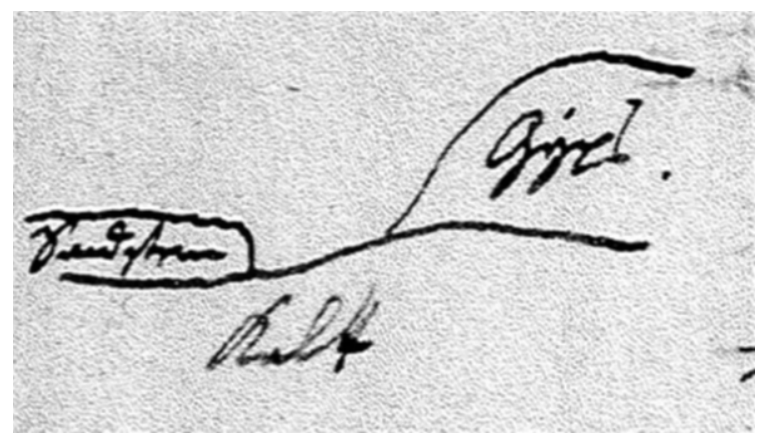

[Sandstein $=$ arenisca, Kalk $=$ roca caliza, Gips $=$ yeso $]$

Valdemoro rodeado de muchos olivos ${ }^{\mathrm{xxvii}}$ (283 toesas). La colina calcárea que circunda el valle del Tajo en Aranjuez y que medí junto con el Sr. Thalacker tiene la misma altura en ambas partes, es decir, que está entallada. Madrid (313 toesas o 1.878 pies sobre el mar). La ciudad está en parte edificada sobre yeso. Éste aflora en el Retiro. Toda la greda alrededor de Madrid así como todas las terrazas son altamente salinas ${ }^{\mathrm{xx}} \mathrm{xii} . / 7 /$

11 H. al margen: il y a aussi du Sel gemme à Mingranilla près Cabriel (La Mancha) et Cardona (Catalogne), véase ANTILLON, I. de (1824), Geografia astronómica, natural y politica de España y Portugal, Madrid, D. Leon Amarita, p. 174. 
[Botánica]

En enero de $1799^{12}$ florecen en los alrededores de Valencia:

Cistus manifolius y Cistus incanus. Bellus perennis. Rosmarinus. Euphorbia serrata - Thymus serpyllum - Viburnum. Opulus - Amygdalus persica. Globularia alypum Daphne - Erica vagans. Erica mediterranea - Calendula officinalis - Sonchus - Clipeola maritima - Sinapis - Passerina - Genista Scorpius Spartium - Narcissus Jonquilla - Helleborus niger - Melocotoneros - Geranium romanum - Guisantes - Judías - Satureja officinalis - Cucurbita - Galium - Polygonum maritimum - Vinca pervinca - Lamium purpureum - Plantago maritima - Salvia - Arbutus unedo - Stachys recta - Lavendula stoechas. Lavendula multifida. Potentilla - Rhamnus alaternus. Lauatera maritima.

Acerca de una observación sobre la suavidad del clima. Olivos por todas partes y muy hermosos en La Mancha a 430 toesas de altitud. En donde faltan nombres específicos de plantas, subsanamos con el libro ${ }^{\mathrm{xxix}}$ de Bonpland.

Comprobado muy exactamente con el cronómetro:

a. Andando muy despacio durante 1 minuto, 115 pasos $=258$ pies parisinos.

b. Andando a paso rápido normal durante 1 minuto, 120 pasos $=270$ pies parisinos.

c. Andando a paso muy rápido durante 1 minuto, 150 pasos $=337$ pies parisinos.

Con la velocidad de $b$ o 4,5 pies en 1" recorro con frecuencia, en un día, 14 leguas francesas, descansando sólo una vez cada hora. Una milla geográfica, es decir, 24.000 pies, se cubren en una hora 28 minutos. Para medir bases grandes de 300 toesas con pasos es muy importante hallar la velocidad con la que se camina de forma uniforme. Cada persona posee una idiosincrasia de su fuerza muscular según su velocidad y en ésta el error es muy pequeño. /8/

\section{Clima}

En el Reino de Valencia el termómetro ya se encontraba, en enero, a $18^{\circ}$ a la sombra y durante varias semanas, desde $12^{\circ}$ a $14^{\circ}$ y sin embargo todos los árboles y arbustos estaban tan deshojados como si estuviera en los $50^{\circ}$. Inclu-

12 H. al margen: entre Barcelona y Valencia. 
so los brotes no se habían desarrollado. Sólo conservan las hojas los árboles que tienen un parénquima apergaminado (Citrus Laurus, Viburnum tinus Olea europaea Chamaerops humilis). En Galicia, en los valles cálidos en donde el tronco del Laurus nobilis alcanza los 40 pies, los Fagus Castanea todavía no tenían hojas el día 26 de mayo. El follaje de los árboles ${ }^{13}$ se introduce en toda España apenas tres semanas antes que en Berlín. Generalmente, todavía a mitad de mayo es cuando se presenta todo reverdecido. ¿Por qué, por ejemplo, en Valencia, los brotes de los chopos, los arces y las hayas no se desarrollan, cuando sin embargo el sol resplandece por espacio de varios meses? ¡Con qué rapidez un día cálido estimula la vegetación en el norte! ¿Le falta a la atmósfera hibernal algo que en la primavera despierte las plantas, como por ejemplo la gran carga eléctrica propia del ambiente primaveral? O quizás, en el sur, las plantas crecen con tanta lentitud o son tan insensibles porque el calor es un estímulo habitual para ellas, porque la savia siempre está en ellas en actividad y porque la misma duración del día les brinda perpetuamente casi ${ }^{14}$ el mismo estímulo solar.

En el norte el sueño hibernal almacena la irritabilidad de las plantas. Está privado del calor del sol a lo largo de casi cuatro meses. La luz de los días largos de verano ${ }^{15}$ produce, después de las largas noches de invierno, la nieve, el aguanieve, que contiene mucho oxígeno disuelto. /9/

España. Madrid, Castilla la Vieja. - León ${ }^{16}$. Galicia

Muy buenas mediciones con el barómetro, con tiempo muy estable.

Madrid 312,2 líneas

El Escorial 297,8.

Guadarrama 300,8.

Puerto de Guadarrama, en donde se encuentra el Alto del León ${ }^{\mathrm{xxx}} 280$.

Villacastín 295,8

Sanchidrián 302,5

Ataquines 309,2

Medina del Campo 312,8

Tordesillas 312,7

13 De los árboles añadido por $H$.

14 casi añadido por $H$.

15 verano añadido por $H$.

16 Castilla la Vieja | Castilla la Nueva tachada | León $H$. 
Venta de Almaraz 309,5

Villalpando 313,5

Benavente 312,8

La Bañeza 310,3

Astorga 308 líneas

por la mañana 306

Puerto Manzanal 296

Bembibre 314

por la mañana 315,2

Villafranca 312

por la mañana 322

Venta del Pagador del Castro 303,5

cumbre de esquisto 296,2

Los Nogales 321,6

Sobrado ${ }^{17}-318$

Lugo - 323

Santiago de Baamonde - 325

Guitiriz - 322,8

Betanzos - 339 .

Inclinación magnética nueva división $\left(\mathrm{en}^{\circ}\right)$
Oscilación

en 1 '
Guadarrama

Sanchidrián

Medina del Campo

Villalpando

Villafranca

Sobrado
73,50

72,30

73,50

73,50

76,15

75,75
24

23,5

24

24

24

24,8

El error es ciertamente muy pequeño cuando se calcula un lugar tras otro como el viaje de París a Marsella, y como enseñan las comparaciones con las mediciones de Deluc y Shuckburg. Aunque todavía estoy esperando cálculos barométricos desde Madrid.

Después ${ }^{18}$ de los montes de granito y sienita y hasta Guadarrama (en for-

17 321,6.| \{ilegible\} 296. tachado | Sobrado $H$.

18 (1) Alrededor (2) Después $H$. 
ma de greda y de tapial como en los montes de Fichtelgebirge; no se sabe si son cantos, bolas o estratificación irregular) se extiende una superficie inmensa hasta Astorga ${ }^{\mathrm{xxxi}}$. En Villalpando, arenisca sobre roca caliza ${ }^{\mathrm{xxxii}}$. En La Nora se hace notorio el gneis de 3,2 horas con $80^{\circ}$ Noroeste xxxiii. En Astorga, gneis de 4 horas suroeste. El Puerto de Manzanal, una sierra de cinco leguas de longitud, muy romántica, de grauvaca $^{\mathrm{xxxiv}}$ y de pizarra grauvacosa de grano fino, finalmente muy fino, es bastante antigua y tiene un aroma a piedra diabasa (afinidad con la arenisca y la diabasa) e inclusiones esféricas con una regularidad de 3-4 horas con $40^{\circ}$ y $70^{\circ}$ noroestexxxv.

En Villafranca, antiguo esquisto arcilloso debajo y hacia fuera, generalmente de 8-9 horas, unas veces al norte otras al sur. Después de Trabadelos se convierte en esquisto micáceo ${ }^{\text {xxvi }}$. En la profundidad, 4 horas norte con bastante regularidad. Formación en valle como en Lauenstein. Mucha hematita en simas y yacimientos de cuarzo ${ }^{\mathrm{xx} x v i i}$.

En la Venta del Pagador de Castro, esquisto micáceo de 3,4 horas con $70^{\circ}$ norte. Contiene muchos depósitos de roca caliza azul, de factura más compacta que granulada (Col de Balme) de 9 horas en vertical ${ }^{\text {xxxviii. }}$.

Antes de Los Nogales hay un gran fragmento de esta roca calcárea con muchas incrustaciones de octaedros de calcita y cristal de cuarzo. Se dirige cada vez más hacia las 11 horas en vertical ${ }^{19 x x x i x}$. Esquisto micáceo. /10/ Después en Sobrado, extensión de 12 horas, no contiene granates sino muchísimos pequeños cubos de pirita de hierro ${ }^{\mathrm{xl}}$.

Tras Sobrado, granate con feldespato grande cristalino. Dentro de un yacimiento inclusiones esféricas de 4-5 brazas, como en Seiffen, con 4 o 6 fragmentos claramente separados ${ }^{x l i}$. Después gneis de 3,5 horas, norte. Después esquisto micáceo de 4 horas sur $85^{\circ}$. En Lugo y Baamonde, gneis y esquisto micáceo con exacta intermitencia de 4 horas norte. Después una gran altiplanicie de granito y el granito estratificado, 2 horas ${ }^{x l i i}$.

En Guitiriz, granito con esquisto micáceo de dos clases, negro y plateado, de 3 horas, solamente en valles, unas veces hacia el sur otras hacia el nortexliii.

Después, el día 28 de mayo, tormenta en La Coruña.

Electrómetro sin yescax ${ }^{x \text { liv }}$, de 2 pulgadas negativo.

Comprobado el aire en el Fucus serratus ${ }^{20}$, mantuvo 0,32 de oxígeno.

$1911 \mid$ gneis tachado | esquisto micáceo $H$.

20 Fucus $\mid$ vericans tachado $\mid$ serratus $H$. 


\section{NOTAS FINALES (COMENTARIOS DE LA EDITORA)}

i «Cerca de Valencia, hay campos cultivados como huertas» (PUIG-SAMPER y ReBOK (2007), p. 189).

ii «Más hacia el sur, roca caliza desnuda, aparentemente la misma formación que reconocí junto a Tarragona, Oropesa y La Mancha y que en el Col de Balaguer está cubierta por nagelflúo» (PUIG-SAMPER y REBOK (2007), p. 189). Nagelflúo $($ Nagelfluh $)=$ conglomerado diluvial de cantos rodados de caliza y otras rocas, presentes como cabezas de clavos en la superficie superior de peñascos. Esta roca suele utilizarse como piedra de mampostería y, cuando está tallada, como revestimiento de fachadas y paredes.

iii «Alcudia, un pueblo en el lugar más encantador, colocado como una joya alrededor de un lugar de peregrinación, (al aire libre) Schinus molle [...]» (PUIG-SAMPER y REBOK (2007), p. 189).

iv 1 toesa $=1,9484 \mathrm{~m}$

$\checkmark$ «Se acerca uno a la cadena montañosa, que en esta zona del sur del reino de Valencia se extiende de Este a Oeste» (PUIG-SAMPER y REBOK (2007), p. 189).

vi «Río Júcar; luego Sierra de S. Ana [...], piedra caliza cubierta por una formación de greda y en ella sobresale un poderoso estrato de yeso» (PUIG-SAMPER y REBOK (2007), p. 189).

vii «Este yeso (que se extiende irregularmente hor. 9,5 y cayendo hacia mediodía $48^{\circ}$ ) granuloso-foliado mezclado con arcilla, muy parecido al que, en Villarrubio y en La Mancha, contiene sal gema» (PUIG-SAMPER y REBOK (2007), p. 189).

viii «El camino asciende suavemente hacia la meseta interior de España. El estrato de yeso aparece particularmente meteorizado, especialmente allí donde está en contacto con piedra caliza, o quizá lo atraviesa. Un valle cultivado entre dos sierras calcáreas y cubiertas de bosques» (PUIG-SAMPER y REBOK (2007), p.189).

ix «La cadena de colinas junto al camino se dirige de SSO a NNE» (PUIG-SAMPER y REBOK (2007), p. 189).

$\mathrm{x} \quad$ «[...] una peculiar descomposición del estrato calizo junto al mojón 51. Cuatro cortes en los que se reconocen con claridad las masas de roca caídas» (HUMBOLDT (1869), p. 189).

xi «[...] después, un monte empinado, Puerto de Almansa [...]. Aquí se llega propiamente a la meseta (plateau), que, casi ininterrumpidamente se prolonga desde La Mancha, a través de la Nueva y Vieja Castilla, hasta el reino de León. La llanura aparece como un antiguo fondo del mar» (PUIG-SAMPER y REBOK (2007), p. 189).

xii «Junto a la ciudad de Almansa, un peñasco calcáreo aislado de apenas 80 pies de altura, cubierto por las ruinas de un antiguo castillo» (PUIG-SAMPER y REBOK (2007), p. 189).

xiii «Otra vez sobre la caliza una pequeña formación de arenisca con cantos rodados de cuarzo, nódulos ferruginosos color marrón y cementos silíceos, probablemente muy diferentes de la formación costera de nagelflúo» (PUIG-SAMPER y REBOK (2007), p. 190).

xiv «Llanuras cerealistas y desarboladas de la provincia de La Mancha, Albacete [...], La Roda [...], Minaya [...]» (PUIG-SAMPER y ReBoK (2007), p. 190).

xv «Aquí la formación calzárea se hace muy porosa, casi como con burbujas, parecida a las calizas del Jura, entre Streitberg y Muggendorf en Franconia» (PUIG-SAMPER y REBOK (2007), p. 190).

xvi «Muchos vetas de roca córnea conchiforme, en transición a la calcedonia, a menudo racemosas, en barras arriñonadas, dispersas en la matriz, probablemente de la formación 
calcárea, yacimiento análogo al de Vallecas, junto a Madrid» (PUIG-SAMPER y REBOK (2007), p. 190).

xvii «Alrededor de Toboso, cuyo nombre ha difundido Cervantes amplia y gloriosamente, se encuentra de nuevo sobre las calizas una formación de arenisca, por lo regular de grano fino, en su mayor parte, compuesto de granos redondos de cuarzo, pero alternando, aquí y allí, con estratos de granos toscos de nagelflúo» (PUIG-SAMPER y ReBOK (2007), p. 190).

xviii «Esta arenisca no parece estar muy extendida y, a causa de la proximidad del granito de Toledo, crecen considerablemente hacia Ocaña los grandes vetas de cuarzo. Los estratos se extienden aquí, como las calizas, por toda la meseta hor. 8-10, y disminuyen, con 8 y 9, bien hacia el norte, bien hacia el sur» (PUIG-SAMPER y REBOK (2007), p. 190).

xix «Alrededor yacimientos artificiales de salitre, montones cónicos de arcilla que, cuando la atmósfera está electrizada, aquí después de una tormenta (como en Cuyavien en Hungría), se cubren de salitre» (PUIG-SAMPER y REBOK (2007), pp. 25-190).

xx HuMBOLDT (1799b), véase también la Introducción.

xxi «Media hora después de Ocaña se entra en el amplio valle del Tajo, que en otros tiempos fue excavado por una immensa corriente, de la que sólo queda un diminuto cauce» (PUIGSAMPER y REBOK (2007), p. 190).

xxii «En el valle mismo, unas pequeñas colinas calizas de apenas 20 toesas de altura, que se elevan erguidas a modo de islas o fortalezas, cautivan la mirada y ofrecen por todas partes estratos caídos» (PUIG-SAMPER y ReBOK (2007), p. 190).

xxiii «Todas las colinas de alrededor de yeso folioso mezclado con arcilla, frecuentamente de 50 brazas (Lachter) de espesor, separadas por estratos calizos. Muchas cavidades (Schlotten) en el yeso» (PUIG-SAMPER y REBOK (2007), p. 191).

xxiv «Parmi les sels que produit l'Espagne, on doit citer, outre mille sources salées, les mines de sel gemme de la Mingranilla, dans la Mancha, près du Cabriel; et celles du Cardona en Catalogne» (ANTILLON (1824), p. 126).

xxv «Esta curiosa formación del yeso de Aranjuez contiene sal gema, junto a Villarrubio, en un valle que he visitado desde Madrid» (PUIG-SAMPER y REBOK (2007), p. 191).

xxvi «En el valle, sobre las calizas, se ha extendido la fomación nagelflúo, bien de grano

basto, bien de grano fino y a menudo calcáreo» (PUIG-SAMPER y REBOK (2007), p. 191).

xxvii «Valdemoro, rodeado de hermosos olivos» (PUIG-SAMPER y REBOK (2007), p. 191).

xxviii «Madrid en parte (en el Retiro) edificado sobre yeso, que, como toda la arcilla de alre-

dedor, tiene algo de sal común» (PUIG-SAMPER y REBOK (2007), p. 191).

xxix Journal botanique, véase la Introducción.

xxx «Puerto de Guadarrama o el León, el famoso puerto del León [...]» (PUIG-SAMPer y REBOK (2007), p. 192).

xxxi «Cuando se desciende hacia el NO de esta cadena granítica que separa como un murallón ambas Castillas, aparece en la bajada Villacastín [...], Sanchidrián [...] y Ataquines [...]. Desde este último lugar se extiende una extensa, casi ininterrumpida, llanura de 30 millas de longitud hasta cerca de Astorga» (PUIG-SAMPER y REBOK (2007), p. 192).

xxxii «Villalpando [...], aquí una formación de arenisca sobre caliza blanca» (PUIG-SAMPER y REBOK (2007), p. 192).

xxxiii «Cerca de Zamora aparece otra vez gneis, extendiéndose hor. 4,3 e inclinación $60^{\circ}$ hacia SO» (PUIG-SAMPER y REBOK (2007), p. 192).

xxxiv Grauvaca (al. Grauwake)= Antiguo término minero germano procedente de graw (gris) y wake (alterado). Piedra arenisca fuertemente compacta de color oscuro, gris 


\section{EL DIARIO DE ALEXANDER VON HUMBOLDT EN ESPAÑA}

o rojizo, impermeable y resistente al fuego. Se trata de una roca detrítica cuyo origen es la descomposición del granito. Tiene mica, feldespato y cuarzo. Su textura es arenosa.

xxxv «Puerto Manzanal [...]. Unas montañas de 5 millas de longitud con románticas quebradas, formadas por grauvaca y especialmente por grauvacas-pizarras de grano muy fino, con extensión hor. 3-4 e inclinación $70^{\circ} \mathrm{NO}$. En esta formación intermedia aparecen inclusiones esféricas, mezcladas en el interior con hornblenda, en transición a diorita» (PUIG-SAMPER y REBOK (2007), p. 193).

xxxvi «En el valle se extraen pizarras arcillosas (hor. 8-9, inclinadas bien al NE, bien al NO) pasando después de Trabadelo a pizarras micáceas» (PUIG-SAMPER y REBOK (2007), p. 193).

xxxvii «Ahí vuelve a ser de nuevo el espesor, como se observó casi en general en esta parte de España, hor. 3-4. Mucha mica en simas y yacimientos de cuarzo» (PUIG-SAMPER y REBOK (2007), p. 193).

xxxviii «Esta venta se encuentra situada en la vertiente sur de una montaña micáceas, cuya pico más alto, al que subí, alcanzaba 580 toesas de elevación. Aquí se llega a un punto, en el que se reconoce de nuevo, lo difícil que es con frecuencia marcar una frontera entre rocas primarias y secundarias. Este pizarra micácea, que pudiera ser considerada como primaria, contiene leves yacimientos de caliza azul, en general de fractura compacta, raramente en transición a granuloso con vestigios de trilobites» (PUIG-SAMPER y REBOK (2007), p. 193).

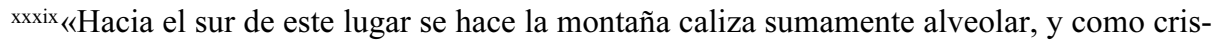
talizada, quizá roca dolomítica, enseguida encima otra vez pizarras micáceas, pero con una extensión muy variable hor. 11-12» (PUIG-SAMPER y REBOK (2007), p. 193).

xl «Ningún rastro de granates, pero cerca de Sobrado [...] muchos cubos de pirita» (PUIGSAMPER y REBOK (2007), p. 193).

xli «Entre Sobrado y Lugo [...] aflora de las pizarras micáceas el granito de grano grueso, en el que observé bolas enormes con fragmentos separados a modo de cáscara. El granito de las bolas es de grano más fino que el de la matriz, pero muy parecido a éste. Este fenómeno geognóstico me recordó vivamente uno muy parecido entre Seiffen y Wunsiedel en el Fichtelgebirge». (PUIG-SAMPER y REBOK (2007), 193). «Enérgicamente asomó en mi alma al recordar que hacía poco tiempo que lo acababa de ver por primera vez cuando estuve en prácticas, en calidad de minero en el territorio de Bayreuth» (HuMBOLDT (1869), p. 30; falta en PUIG-SAMPER y REBOK).

xlii «Desde aquí hasta el límite noroeste del granito aparecen primero gneis, después pizarras micáceas, y pronto junto a Lugo [...] y Baamonde [...] gneis alternándose con pizarras micáceas; todos discurriendo con gran regularidad, como la alineación montañosa misma, de SO a NE» PUIG-SAMPER y REBOK (2007), p. 193).

xliii «Todo el declive entre Betanzos y La Coruña es otra vez una auténtica meseta de granito con mica de dos clases, marrón y plateada. Aquí y allá se encuentra el granito granuloso, claramente estratificado (sin tránsito a gneis), formando franjas hor. 2; sólamente en los valles más profundos aflora algo de pizarra micácea» (PUIG-SAMPER y REBOK (2007), p. 193).

xliv Yesca. Para el método voltaico se utilizó humo en la aplicación del electrómetro, véase la Introducción.

Recibido: 9 de junio de 2008.

Aceptado: 10 de junio de 2008. 